\title{
Miradas actuales sobre jóvenes, estudio y trabajo. Un recorrido epistemológico
}

Current views on youth study and work. An epistemological journey

Mariel Ayelén Neme ${ }^{1}$

\section{Resumen}

Este trabajo propone un recorrido por una serie de investigaciones que se ocuparon de indagar los sentidos que los jóvenes otorgan al estudio y al trabajo. Si bien no podría decirse que constituye un "estado del arte" porque seguramente han quedado excluidos múltiples análisis valiosos en relación al tema, si podría afirmarse que consiste en una sistematización de algunas producciones. Éstas han sido seleccionadas desde un recorte temporal, ya que se abarca autores que se dedicaron a estudiar el tema a partir de los años 90 , geográfico, porque principalmente se trata de investigaciones latinoamericanas y disciplinar, ya que los estudios provienen especialmente de la sociología, la educación y la antropología. Sin embargo, quizá la característica principal que comparten las investigaciones aquí seleccionadas es que poseen una misma perspectiva epistemológica: la consideración de la categoría juventud como una construcción social. De este modo, los estudios reunidos en este artículo piensan a las juventudes en plural, considerando que "ser joven" se define en relación con una multiplicidad de personas, grupos, actores, colectivos cuyas características y propiedades no se agotan en descripciones homogeneizantes sobre la juventud, sino que destacan la multiplicidad de contextos, actores y las maneras en que las juventudes son producidas.

Las producciones aquí presentes invitan a la reflexión, a abrir el diálogo entre quienes hoy están produciendo conocimiento en la región y pretende ser un aporte para continuar reflexionando desde el intercambio y el diálogo con otros.

Palabras Clave: jóvenes, educación, trabajo.

\section{Abstract}

This work proposes a journey through a series of investigations that were dedicated explore the senses that young people give to study and work. Although it could not be said that it constitutes a "state of the art" because surely many valuable analyzes

Recibido: 24 de marzo de 2019 Aceptado: 2 de septiembre de 2019 Publicado: 20 de diciembre de 2019

${ }^{1}$ Maestranda en Sociedad e Instituciones (FCEJS-UNSL). Licenciada en Comunicación Social. Docente e investigadora de la Facultad de Ciencias Económicas, Jurídicas y Sociales. Universidad Nacional de San Luis (FCEJS-UNSL). Correo electrónico: ayeneme@hotmail.com 
have been excluded in relation to the subject, if it could be affirmed that it consists of a systematization of some productions. These have been selected from a temporal cut, since it covers authors who were dedicated to studying the subject from the 90s, geographically, because mainly it is about Latin American research and discipline, since the studies come especially from sociology, education and anthropology. However, perhaps the main feature shared by the research selected here is that they have the same epistemological perspective: the consideration of the youth category as a social construction. In this way, the studies gathered in this article think of the youth in plural, considering that "being young" is defined in relation to a multiplicity of people, groups, actors, collectives whose characteristics and properties are not exhausted in homogenizing descriptions about youth they highlight the multiplicity of contexts, actors and the ways in which youths are produced. The productions presented here invite to reflection, to open dialogue between those who are now producing knowledge in the region and intends to be a contribution to continue reflecting from the exchange and dialogue with others.

Keywords: youth, education, work.

\section{Introducción}

Desde la década del 90, a partir de los diversos cambios mundiales en el plano social, económico y político, un gran número de investigaciones sobre juventudes se ocuparon de analizar el significado que éstas le atribuyen a la escuela y al trabajo, dos instituciones propias de la modernidad que, en la actualidad, se redefinen; y, por lo tanto, son puestas en cuestionamiento e instan a renovar la mirada y el análisis.

En la Argentina la mayoría de las investigaciones sobre jóvenes en relación a la educación y el trabajo (Chaves 2006), han sido analizadas desde la perspectiva de la inclusión-exclusión y desde una diversidad de enfoques analíticos (aunque predomina una perspectiva crítica), metodológicos (estudios estadísticos, etnográficos, etcétera), ejes de conocimiento (desde cuestiones pedagógicas hasta macro estructurales) y de referente empírico.

En un intento de integración de estos antecedentes, este artículo propone un recorrido por una selección de diversos estudios, especialmente latinoamericanos, que se ocuparon de indagar en esta relación a fin de profundizar en el conocimiento del tema de referencia y reflexionar sobre la epistemología existente. Las investigaciones seleccionadas provienen de diversas disciplinas principalmente de la educación, la psicología y la sociología; aunque seguramente habrán quedado en el 
camino muchos otros estudios interesantes y complementarios que no han sido incluidos en este trabajo.

En un intento de integración y de considerar los aportes de los diferentes autores, se ha estructurado el artículo en tres grandes apartados: "Juventudes en plural", "Lxs jóvenes en la institución educativa, Jóvenes y mundo laboral”. El primer apartado "Juventudes en plural" propone un recorrido por los diferentes estudios que marcaron una ruptura epistemológica ya que no pensaron la categoría "juventud" como un todo homogéneo, sino que consideraron que existen diferentes formas de transitar la juventud, perspectiva epistemológica que atraviesa este artículo. Así, este conjunto de investigaciones hace hincapié especialmente en los sentidos otorgados por lxs jóvenes a los espacios sociales y culturales por los que transitan y analizan las subjetividades y representaciones que se articulan de modo diferente con el todo social.

"Lxs jóvenes en la institución educativa" está formado por investigaciones referidas a lxs jóvenes en la escuela media a partir de las diversas transformaciones del sistema educativo argentino que fueron reconfigurando su fisonomía. Así, estas investigaciones analizan tensiones desde las coordenadas actuales, a partir de las formas de ser joven, la desigualdad y fragmentación educativa, las trayectorias escolares y el sentido que lxs actores otorgan a su tránsito por la escuela. Se presentan en este apartado estudios latinoamericanos referidos a la temática y hacia el final se incorporan investigaciones especialmente de Argentina. Este conjunto de estudios sobre jóvenes y escuela media constituyen un corpus importante desde la sociología, la educación y la pedagogía que aportan al debate sobre jóvenes, estudio y trabajo en relación al contexto de la escuela media a la que asisten.

El tercer apartado "Jóvenes y mundo laboral” agrupa investigaciones concernientes al mundo del trabajo. Por un lado, un conjunto de estudios se enfoca en los sentidos dados por los jóvenes al trabajo de acuerdo a su edad cronológica y los diversos procesos que atraviesan en sus vidas y otros enfocados en las trayectorias laborales juveniles en relación a sus propias experiencias y transiciones. Estas investigaciones permiten reflexionar sobre las experiencias comunes y diferentes que se constituyen en torno al trabajo en los diferentes contextos de los cuales los jóvenes forman parte.

Es preciso reiterar que, como se aclaró al inicio del artículo, en este escrito se ha intentado obtener, seleccionar y sistematizar aquella información relevante que de un modo significativo aporta a la comprensión del corpus existente en la relación de jóvenes, estudio y trabajo, aunque seguramente habrán quedado en el camino muchos otros estudios interesantes y complementarios que no han sido incluidos. 


\section{Juventudes en plural}

Uno de los investigadores precursores en considerar a la juventud como una categoría heterogénea es Bourdieu, que ya en 1978 publicó su libro "La juventud no es más que una palabra” en el que cuestiona las categorías de jóvenes y adultos aduciendo que las mismas se construyen socialmente. Por otro lado, propone considerar la categoría juventud en plural distinguiendo entre los jóvenes estudiantes y aquellos de la misma edad biológica que ya trabajan.

En los últimos años se han producido fructíferos análisis sobre la pertinencia del criterio etario, en el sentido de pertenecer un rango de edad, para configurar a los jóvenes como categoría analítica (Bourdieu, 1990; Margulis y Urresti 2000; Wortman, 2001; Margulis 2002; Urresti 2002).

En lo que respecta a la Argentina, Braslavsky (1986) organiza una clasificación de los discursos sobre la juventud en el mencionado país y propone una perspectiva de heterogeneidad al analizar metafóricamente a la juventud dorada, gris y blanca aduciendo que el mito de la juventud homogénea es un mecanismo por el cual se identifica a todos los jóvenes con algunos de ellos. La misma autora en 1989 analiza los estudios realizados desde 1983 sobre juventud en América Latina y el Caribe y plantea que uno de los principales aportes del conjunto de estudios e investigaciones sobre el tema consiste en haber desmitificado definitivamente la idea de la existencia de una juventud en América Latina. "La juventud de cada país es muy diferente a la de los demás, y al interior de cada nación los estilos de inserción y las modalidades de acción de los jóvenes varían notablemente" (Braslavsky, 1989:32)

Por su parte, y también en Argentina, Mario Margullis en su libro "La juventud es más que una palabra” editado en 1996, destaca los sentidos singulares y usos particulares que denota el término juventud, destacando la referencia a la juventud con la multiplicidad de situaciones sociales en que esta etapa de la vida se desenvuelve y planteando la necesidad de hacer explícitos los marcos sociales históricamente desarrollados que condicionan las distintas maneras de ser joven.

Respecto a la concepción de juventudes en relación a las grandes instituciones de la modernidad como son el Estado, la familia, la escuela y el trabajo, Reguillo (2000) advierte que la juventud, es una construcción de posguerra, por cuanto las sociedades del "primer mundo" identifican al joven no sólo como sujeto de derecho sino como sujeto de consumo. Plantea que, con algunas excepciones, el Estado, la familia y la escuela siguen considerando a la juventud como una categoría de tránsito entre un estado y otro, una etapa de preparación para lo que la juventud vale como futuro. Mientras que, para los jóvenes, su ser y su hacer en el mundo está anclado en el presente, lo que ha sido finamente captado por el mercado. 
Desde un análisis de la concepción de juventudes en relación al mercado Urcola (2003) afirma que, se considera a la juventud como una construcción histórica ligada a las necesidades de las fuerzas productivas en los comienzos de la Revolución Industrial con la necesidad de mayor capacitación de las futuras generaciones durante un período que oscila entre la niñez y la adultez. Plantea que lo que se produce es un ajuste en la cosmovisión del concepto. Mientras que en los tiempos precedentes la juventud se sustentaba bajo los emblemas de la valentía, la fuerza y la voluntad transformadora, en los tiempos modernos se produce un ajuste de estos atributos hacia el campo de la producción (mundo del trabajo) y posteriormente hacia el mercado de consumo (consumismo) y advierte que en la modernidad la idea misma de madurez social está asociada al ingreso al mercado laboral y asunción de obligaciones y responsabilidades civiles.

Teniendo en cuenta la adhesión a los significados y consumos culturales como forma de identificación social y diferenciación del resto del colectivo social, existe una cultura de lo juvenil (Urcola, 2003) El campo cultural, desde las manifestaciones culturales o contraculturales se convierte en el ámbito de identificación juvenil.

Por su parte Feixa y Porzio (2004) definen a las culturas juveniles en dos sentidos. Por un lado, desde un punto de vista genérico a partir del cual las experiencias sociales de los jóvenes son expresadas colectivamente mediante la construcción de diversos estilos de vida, localizados fundamentalmente en el tiempo libre o en espacios intersticiales de la vida institucional. Por otro lado, desde una perspectiva restringida, en la cual se hace referencia a la aparición de micro sociedades juveniles, con grados significativos de autonomía respecto de las instituciones adultas, que se dotan de espacios y tiempos específicos, y que se configuran históricamente en los países occidentales tras la II Guerra Mundial.

Más allá de las diferencias históricas, sociales, culturales y las diversas perspectivas de conocimiento, estas investigaciones tienen en común el considerar a lo juvenil como una condición social, abordando la existencia de "juventudes" desde una perspectiva polisémica, que reconoce que las distintas formas en que se expresa lo juvenil constituyen la expresión de diferentes formas de vivir, sentir y dar sentido a la condición juvenil desde los distintos grupos. En este sentido, Joaquín Casal (2006) propone entender las juventudes desde un enfoque sobre itinerarios y transiciones basado en la crítica a la linealidad de los procesos de incorporación a la vida adulta, propios del período industrial, asimismo plantea una crítica sobre el auge de perspectivas basadas en la reversibilidad de las transiciones, señalando la complejidad y los efectos del capitalismo informacional en los jóvenes. En el mismo sentido Oscar Dávila León (2008) analiza las trayectorias y transiciones juveniles analizando por un lado el impacto de los procesos educativos en las trayectorias 
juveniles y las posibilidades de acceder a una condición satisfactoria de inserción social.

Estos estudios permitieron tener en cuenta diversos aspectos que constituyen la perspectiva epistemológica que atraviesa el artículo. Por un lado, desactivar la mirada adultocéntrica (Chaves, 2006) por otra parte plantear múltiples ejes para el abordaje de las juventud en plural, por fuera del mito de su homogeneidad (Braslavsky, 1986) y principalmente escuchar las voces de los jóvenes y reconocer sus resistencias y acciones más allá de los marcos formales (Reguillo, 2000) a fin de conocer sus experiencias (Núñez y Lietichiever, 2015) y detectar nuevos modos de participación (Kropff y Núñez, 2008 Saintout, 2006 y 2010)

Atentos a las nuevas construcciones que adquiere la juventud como categoría analítica es necesario indagar en las particularidades, la multiplicidad de historias y realidades, entre contradictorias y afines, por las que atraviesan. En este sentido adquiere relevancia el análisis de las prácticas juveniles y por tanto cobra importancia en el análisis la relación de los jóvenes con la educación y el trabajo.

\section{Lxs jóvenes en la institución educativa}

A partir de los diversos cambios estructurales ocurridos a lo largo del tiempo en la institución escolar, una gran parte de las investigaciones se han enfocado principalmente en el análisis de la fragmentación y desigualdad que conviven en el sistema educativo a partir de la incorporación diversificada y desigual de los niños y jóvenes.

Ya en el retorno de la democracia, Braslavsky (1986) señaló la existencia de circuitos diferenciados de educación en coincidencia con el origen social de lxs jóvenes estudiantes, marcando que por lo tanto no solo hay que garantizar el nivel de acceso igualitario para todos sino también implementar diversas estrategias compensatorias para equilibrar los puntos de partida diferentes para el logro de los aprendizajes. La investigación de Duschatzky (1999) representa el primer antecedente clave en preguntarse por la función y sentido de la escuela para los estudiantes de barrios pobres. Este trabajo tiene la virtud de dejar establecida la necesidad de escuchar a los actores escolares para entender la escuela y lo que hace falta en ella. Además, sus resultados arrojan una pregunta poco abordada respecto a la relación entre ser joven y ser alumno, preguntándose si la pertenencia a la institución otorga alternativas de construcción de "lo juvenil".

En lo que respecta al nivel secundario, a partir de los cambios ocurridos en la Argentina en la década de los 90 -como fueron la transferencia de las escuelas a las provincias (Ley 24.048/91) y la Nueva Ley Federal de Educación (24.193/93)- una 
gran literatura en este período se orientó al análisis de estas reformas en relación a la inclusión/exclusión y a la segmentación educativa (Puigróss y Dussel, 1999; Braslavsky1995. Tiramonti; Braslavsky y Filmus 1995)

Este debate continuó a lo largo de los años y se profundizó luego de la sanción en 2006 de la Nueva Ley de Educación que estableció la obligatoriedad del nivel y otorgó al Estado la responsabilidad generar las condiciones para que todos los jóvenes puedan concurrir y permanecer en la escuela. Esta concepción implicó entender la educación como un derecho y significó la consolidación del Estado como garante de una tendencia de masificación del nivel. Esta situación otorgó a la escuela secundaria un nuevo desafío, el de combinar la expansión de la matrícula con una escuela inclusiva y de calidad en un momento de reconfiguración de los roles institucionales, o dicho de otra forma el desafío de la masificación del nivel en un marco de sociedad fragmentada

En Argentina, Inés Dussel(2004) preparó un documento para el Ministerio de Educación de la Nación en el que reflexiona sobre la situación de la desigualdad social educativa en la Argentina presentando un mapeo de la situación educativa, de las propuestas de acción llevadas desde el Ministerio de Educación de la Nación e instando a la necesidad de una verdadera reforma del sistema escolar repensando la idea de igualdad homogénea e incorporando formas de igualdad más complejas dinámicas y rurales.

Luego de la sanción de la nueva Ley Nacional de Educación en el año 2006 el debate por la igualdad educativa siguió siendo centro de debate de los cientistas sociales. En este sentido es valioso el aporte de Tiramonti (2009) quien alude al mito de la sociedad igualitaria y de una escuela pública que incorpora a todos por igual pero que en realidad tiene un rasgo desigual en la medida en que la incorporación se realiza generando segmentos diferentes dentro de los jóvenes que acceden al sistema educativo. Esta fragmentación planteada por la autora resulta de una práctica social que tiende a la segregación y diferenciación de grupos sociales distintos ya sea por una voluntad de separarse para distinguirse de la intromisión de otros o como reacción a la estigmatización de ajenidad o extrañeza social que se proyecta sobre ellos. Dubet (2006) planteó una tendencia similar en Francia ante una democracia que preservó e incluso fomentó las brechas de composición social entre los diversos establecimientos educativos. También en América Latina algunos estudios buscan describir los problemas de equidad y calidad educativa que se observan en los países latinoamericanos (Miranda López, 2012)

Estos estudios tienen en común el análisis de lxs jóvenes y la escuela secundaria a partir de las transformaciones y demandas que recaen sobre este nivel educativo 
haciendo referencia la incorporación diversificada y desigual de los alumnos y la fragmentación que convive en el sistema escolar.

Tenti Fanfani (2000), por su parte, muestra cómo en sus orígenes la escuela media era la antesala de los estudios universitarios y ciertos cargos públicos, por lo tanto, estaba reservada mayoritariamente a los hijos de las clases dominantes. Actualmente, señala el autor, conserva el valor de ser un paso previo y necesario a los estudios superiores, pero en muchos casos es el último escalón educativo al que se accede y se aspira. En paralelo, la devaluación de los títulos vacía de sentido la experiencia escolar tal como era conocida, especialmente por los más bajos sectores, como herramienta de ingreso al mundo laboral y protección ante el desempleo.

Feijoó y Corbetta (2004) se centran en contextos de pobreza y, allí, en la voz de las familias y de los equipos docentes. Dentro del espacio reducido otorgado a los y las jóvenes es interesante la incorporación de chicos desescolarizados (aunque este se encuentre opacado por el de los escolarizados) y el significado que ellos mismos atribuyen a esa condición.

Otro grupo de estudios señalan que las desigualdades educativas no se limitan a un problema de acceso a la educación, sino que adicionalmente, tal como señala Puiggrós (2003 en Córica 2012), la segmentación y la calidad educativa acentúan y consolidan la diferenciación de clases sociales.

En relación a la fragmentación, Kessler (2014) plantea que existen dos tendencias la primera es un aumento de cobertura, cuyo correlato es la desigualdad en lo que refiere a la calidad educativa. En segundo lugar, se presentan desigualdades al interior del sistema, con la conformación de circuitos educativos por clase social que provocan la segmentación del mismo, con una diferenciación creciente entre el sistema público y privado. Además el autor refiere a que, si bien es indudable el incremento de recursos al sistema educativo, la diferencias entre las provincias de mayor población y las más pobres son las que presentan el menor presupuesto para educación y los salarios docentes más bajos.

Algunas investigaciones proponen pensar la construcción de la igualdad más allá de la situación ocupacional o la escuela a la que se asista (Dubet, 2011) En esta línea el estudio de Nóbile (2014) hace hincapié en los vínculos entre docentes y alumnos a partir de que los primeros ponen en suspenso la mirada estigmatizadora de los estudiantes lo que permite relaciones de mayor confianza.

Entre los escasos trabajos que toman la voz de los jóvenes desertores o desescolarizados, vale mencionar también el trabajo de Auyero (1993) y, más cercano en el tiempo, el de Jacinto (2006) que se aboca a la tensión escuela-trabajo. Entre los resultados sobresale la opinión de lxs chicos respecto de que en la escuela no se aprende nada preciso, pero sí a hablar, comunicarse y relacionarse. Ellxs 
consideran que esto les sirve, en términos muy generales, "para el trabajo y para la vida", pero la autora encuentra que no hay una valoración de la experiencia escolar en términos de relaciones, pertenencia, contenido. A la hora de salir a buscar empleo "el título sirve, pero no alcanza" (Jacinto, 2006) de modo que el sentido de la escuela como intermedio hacia el trabajo ya no es suficiente (Zattara y Skoumal, 2008) y, sin embargo, en muchos casos se sostiene la experiencia escolar a pesar de las dificultades. Auyero (1993) encuentra que la escuela para los jóvenes pobres es una escuela para que no les mientan, los engañen, los estafen. La escuela aparece como la institución que brinda la posibilidad de defensa

Chaves, Fuentes y Vecino (2016) analizan las representaciones se sostienen en torno al valor que lxs jóvenes le dan a la escuela secundaria en distintos escenarios de interacción en el que se generan desigualdades

Otro grupo de investigaciones están focalizadas en las trayectorias de lxs jóvenes que transitan la escuela. Algunas destacan que actualmente existe una mayor retención del sistema, pero se incrementan los índices de repitencia y sobre edad aunque disminuye el número de jóvenes que deja la escuela (Montes, 2010) En este sentido Flavia Terigi (2007) hace una interesante distinción entre trayectoria teórica y real. Mientras que la primera sería la esperada, sigue una proyección lineal marcada por una periodización estándar, la segunda está formada por los modos reales en la que los jóvenes transitan su escolarización. La misma autora en el año 2010 propone el concepto "Cronologías del aprendizaje” para plantear que el saber sobre la enseñanza está planteada desde un aprendizaje monocrónico ${ }^{2}$, desde un sujeto construido a la medida de una trayectoria teórica lo cual genera un desacople entre la realidad de lxs estudiantes y la escuela.

En este marco una serie de investigaciones latinoamericanas analizan las trayectorias de jóvenes apelando al concepto de escuelas acotadas que compiten con la temprana incorporación de los jóvenes al mundo del trabajo (Saraví 2010), y el sentido que los jóvenes otorgan a la coexistencia de diferencias y desigualdades socioeconómicas. ( Idem, 2015) Según el autor el tiempo escolar está restringido a lo que ocurra en la escuela dado que muchos jóvenes no cuentan con espacio ni tiempo en el que puedan realizar las tareas o estudiar y tampoco con el apoyo de su familia. Este grupo de investigaciones ayudan a pensar en que muchos jóvenes en la actualidad, a partir de la obligatoriedad del nivel, están atravesando trayectorias inaugurales en el sentido de ser los primeros en su familia o en sus vínculos cercanos que se encuentran en la escuela secundaria. Por otra parte, sus trayectorias suelen ser discontinuas, ya que especialmente quienes poseen una situación

\footnotetext{
${ }^{2}$ La autora utiliza este concepto en relación al tiempo, es decir la idea de que es necesario proponer una secuencia única de aprendizaje para todos los miembros del grupo escolar
} 
socioeconómica baja, tienen dificultades para asistir a la escuela, ya sea por tener que cuidar a sus hermanos, por embarazo adolescente o por tener que trabajar para ayudar a sus padres. En este sentido cobran gran importancia los estudios citados ya que por medio del análisis cualitativo muestran las experiencias particulares por los cuales atraviesan los jóvenes. En relación a lo anterior, un aporte importante realizan los estudios que proponen pensar la nueva configuración de la educación a partir de experiencias juveniles heterogéneas y analizan los diferentes sentidos que lxs jóvenes le otorgan a la escuela secundaria (Núñez y Litichever, 2015) En este grupo pueden incluirse una serie de investigaciones dedicadas al análisis de las expectativas de los jóvenes al momento de terminar la escuela, e indagan sobre el lugar de las decisiones en jóvenes próximos a egresar de los bachilleratos populares (Mereñuk,2010). En igual sentido Vecino y Guevara (2014) analizaron las expectativas de los jóvenes en la escuela y señalan que existe en los estudiantes una mirada en la que se sostiene que la escuela los preparará para el futuro para estar mejor posicionados ante ciertas condiciones sociales heredadas del contexto que los tocó vivir. Desde el presente la experiencia escolar también es considerada como ámbito de valorización entre pares y de intercambio generacional a través del vínculo particular que supone el espacio escolar. Estas formas de significar el paso por la escuela podrían aludir a una redefinición del sentido que los jóvenes otorgan a la temporalidad. En este sentido la experiencia de lxs jóvenes en la escuela sería importante en el presente porque sería el pasaporte para un buen futuro. Dentro de este grupo de investigaciones pueden ubicarse aquellas que abordan la relación entre educación y género buscando comprender de qué manera dichas relaciones se encuentran presentes e inciden en la construcción de las diversas subjetividades juveniles (Millenaar, 2017)

Este grupo de investigaciones aportaron al debate para analizar tensiones y conflictos que conviven en las diversas experiencias juveniles. Otro grupo de trabajos de gran aporte a esta temática se abocaron en analizar las experiencias juveniles en relación a las características de las escuelas en cuanto a modalidad, formato y localización espacial. Todas éstas son variables desde las cuales se analizará el contexto institucional de este trabajo de tesis. Estas investigaciones se enfocan principalmente en jóvenes de nivel socioeconómico medio y bajo como aquellos que indagan sobre las características de las escuelas técnicas y los motivos por los cuales los jóvenes acceden a ella (Dussel y Pineau 2003), las experiencias de los jóvenes de bachilleratos populares y el acercamiento de sectores que la escuela tradicional no lograba incluir (Gluz, 2013), la educación popular y los sentidos escolares hegemónicos que se tensionan entre militantes y docentes (García,2016) y 
en menor medida existen estudios dedicados a investigar la experiencia escolar en sectores de elite(Ziegler,2011).

La variable de género ha tenido un particular abordaje en los trabajos que abordan la experiencia escolar. La mayor parte de las investigaciones (Redondo, 2004; Duschatzky, 2000; Ruiz 2004) entienden al género como una categoría que a la luz de los resultados amerita ser señalada diferencialmente, pero que no había sido incluida con anterioridad como variable de importancia en el estudio. Por otra parte, hay una serie de trabajos centrados específicamente en la dupla género y escuela. Aquí es posible clasificar dos nuevos grupos. De un lado, ubicamos estudios como el de Morgade (2001) que focaliza en el problema del currículum oculto o el de Bonder (1994) atento a analizar la igualdad de oportunidades educativas para hombres y mujeres. Ambos focalizan su mirada en la escuela y en cómo desde ella se plantean relaciones diferenciales para unos y otras.

Del otro lado, encontramos trabajos que se preguntan por esta relación, pero recuperando la perspectiva de los jóvenes. En este grupo destacamos la indagación desarrollada por Zattara y Skoumal (2008), quienes se preguntan si los sentidos otorgados a la experiencia escolar difieren según el género en tanto y en cuanto las significaciones van asociadas a los roles y expectativas socialmente construidos sobre varones y mujeres, que la escuela misma contribuye a reproducir. Las autoras sostienen que la escuela aparece como un tiempo y espacio en que se puede ser joven, apartándose momentáneamente de las actividades y responsabilidades más asociadas al mundo adulto en las que se ven involucrados los jóvenes en contextos de pobreza.

\section{Jóvenes y mundo laboral}

El trabajo es una categoría polisémica; y por lo tanto todos los estudios que la abordan comienzan planteando que existen múltiples interpretaciones, definiciones, y aspectos desde el cual analizarlo. Algunos estudios se abocan en analizar la importancia del trabajo en perspectiva histórica en tanto soporte de protección social (Castel, 1997; Grassi y Danani, 2009; Andrenacci y Soldano, 2006; Sennet, 2010; Dubet, 2011; Merklen, 2010). Un grupo de investigaciones analizan las transformaciones ocurridas en el trabajo a partir de los cambios sociales. En este sentido Carlos La Serna (2010:11) afirma que:

La transformación del trabajo da cuenta, como quizá ninguna otra institución de la modernidad, de los procesos políticos, económicos y culturales que la contextualizan. Es el resultado, y en ocasiones también la causa, de cambios en los derechos civiles y políticos, y en las formas de ejercerlos y promoverlos; de transformaciones 
tecnológicas a veces bruscas en los procesos productivos, y en el funcionamiento de los mercados; de mutaciones en las capacidades y modalidades de interpretación individual y social sobre la realidad.

En este punto radica la importancia del análisis de la relación de lxs jóvenes con el trabajo ya que, como sostiene la investigadora Mariela Macri (2010), el trabajo constituiría un medio de expresión personal para los sujetos siendo éste estructurante de relaciones sociales.

Otro grupo de investigaciones ponen el acento en el lugar del trabajo en la construcción de la identidad (Battistini, 2004; Bialakowsky, 2004; Abal Medina, 2004; Busso y Gorban, 2004).

Un número considerable de cientistas sociales han ligado sus investigaciones específicamente al cruce entre juventudes y trabajo (Kessler, 2010; Epele, 2010; Longo, 2004; Silba, 2006; Medán, 2012; Romero, 2012; Meradi, 2009, Córica 2010, Macri 2010) Este apartado retoma las discusiones actuales planteadas en el marco de estas investigaciones para reflexionar sobre las percepciones que se construyen y redefinen en torno al mismo.

El panorama de creciente deterioro del esquema laboral en paralelo al incremento de la escolarización producida a lo largo de las últimas décadas, conforman dos claves centrales que atraviesan los estudios sobre el tema. En este contexto de cambios estructurales en la educación y el trabajo el papel de ambos en relación a las experiencias juveniles traza el recorrido de este eje para comprender las representaciones actuales que los jóvenes definen y redefinen en la contemporaneidad.

Una gran parte de las investigaciones se han orientado a las percepciones que los jóvenes tienen sobre el trabajo en el contexto educativo. Algunos investigadores estudiaron perspectivas y estrategias de jóvenes de escuela media que estudian y trabajan simultáneamente (Macri, 2010). Un aporte significativo en la reflexión sobre este vínculo es el de la investigadora argentina Agustina Córica (2012) quien, considerando a la juventud en cuanto construcción socio histórica, aborda la relación de la escuela media y el mundo del trabajo a partir del análisis de las oportunidades y los condicionantes que intervienen en las decisiones del futuro de los jóvenes estudiantes. De esta forma señala por un lado que las reformas económicas implementadas en las últimas décadas del siglo XX tuvieron como consecuencia el aumento de los niveles de desigualdad y exclusión social en Argentina, haciendo que la situación social y económica del país se tornase crítica. En un recorrido histórico la autora señala que a principios del siglo XXI la situación comenzó a revertirse y lentamente se notan mejoras en la calidad de vida de la población, es así que en el período 2003-2008, la Argentina experimentó un 
crecimiento sostenido, que le permitió recuperarse de una crisis económica profunda y reactivar su economía considerablemente. Según la autora estas transformaciones repercutieron directamente en la inserción laboral general pero principalmente en la población joven quienes cada vez más atraviesan experiencias laborales dispersas, no acumulativas para una misma rama o actividad sino relacionadas con sus trayectos de vida, con los hitos o pasajes que hacen que esta relación sea dinámica.

En este grupo puede incluirse también el trabajo de Dursi y Millenaar (2012) referida a jóvenes de bajo nivel socioeconómico que se esfuerzan por completar su titulación secundaria y participan en capacitaciones como una manera de sumar recursos a su título secundario y mejorar sus capacidades de inserción laboral.

Algunas investigaciones tienen como problemática central la vinculación entre escuelas y empresas ante las exigencias crecientes que deben enfrentar los jóvenes al egresar de la escuela media frente al actual contexto de globalización y competitividad del mundo laboral (Corti,2000) Dentro de esta literatura puede incluirse las investigaciones de Ana Miranda(2008)quien destaca que las tendencias hacia la desigualdad educativa y laboral de los jóvenes de nuestros días se lleva a cabo en un contexto de desestructuración de colectivos e instituciones sociales, es decir un proceso que tiende a la individualización. Analía Otero (2009) quien se ocupó de analizar las transiciones a la vida adulta de jóvenes egresados de diversas escuelas secundarias a fin de conocer sus percepciones sobre educación, trabajo y familia a partir de las actividades que realizan en la actualidad a fin de comprender articulaciones y tensiones.

Estos estudios tienen en común el debate sobre las fuertes desigualdades en las oportunidades de acceso a trabajos formales y estables, a la precariedad laboral juvenil y a los esfuerzos que muchos jóvenes hacen para lograr mejores condiciones de vida. Sus aportes son primordiales en esta tesis ya que sin perder de vista el actual contexto de globalización por el que atraviesan lxs jóvenes proponen el análisis de realidades particulares insertas en diversos contextos sociales, económicos y culturales contemplando las particularidades de cada joven y su entorno social.

Una importante cantidad de trabajos parten del análisis de la experiencia juvenil en el trabajo a partir de las narrativas de los propios jóvenes. Estas investigaciones destacan principalmente la importancia que éstos brindan al trabajo como estructurador de la identidad. Agulló (1998) investigó la centralidad del trabajo en el proceso de construcción de la identidad de los jóvenes aduciendo que las situaciones de desempleo y precariedad laboral repercutía en ellos mediante una serie de fenómenos negativos para la cristalización efectiva de su identidad. 
En esta línea algunos investigadores analizan la heterogeneidad de experiencias laborales que atraviesan los jóvenes tratando de explicar la relación que existe entre éstas, y las actitudes individuales y colectivas de esos jóvenes trabajadores en relación con su trabajo. (Bono y Bulloni, 2008).

Existe una profusa literatura abocada al análisis de los jóvenes y el trabajo desde una dimensión temporal, esto implica el análisis de sus trayectorias. Son numerosas las investigaciones sobre trayectorias (Bourdieu, 1970; Dávila, Ghiardo y Medrano, 2007; Jacinto, 2009; Aisenson, 2007 y Landini, 2009, Torillo, 2010; Torillo y Macri, 2012 entre otrxs autores), todas remiten a la consideración de los procesos vitales de los actores sociales en relación con las estructuras sociales. Ahora bien, tal como lo señala Jacinto (2016) existe una larga tradición académica latinoamericana en investigaciones sobre educación y trabajo, basada en estudios que relacionan directamente las credenciales educativas con la inserción ocupacional de los trabajadores, especialmente con sus ingresos y sus categorías socio-profesionales. Muchos de estos trabajos están referenciados en teorías del capital humano al buscar relaciones entre educación y empleos o entre niveles de escolaridad y desarrollo.

Investigaciones recientes destacan que los tránsitos y las trayectorias de los jóvenes se presentan desestructuradas y diversificadas .(Gary Polock (2002); Pérez Islas (2002); Reguillo Cruz (2000); Machado País (2009); Casal (2006); Biggart (2002).

En América Latina las investigaciones de Gonzalo Saraví (2010) sobre las transiciones de los jóvenes de sectores populares, constituyen un interesante punto de partida para repensar el vínculo entre transición y movilidad social ascendente. Este autor señala la vulnerabilidad de las transiciones de jóvenes que acumulan desventajas sociales, mostrando caminos que conducen a la exclusión como contrapartida de la crisis de las instituciones tradicionales de integración social de los jóvenes tales como el mercado de trabajo.

En lo que respecta a estudios argentinos, Jacinto (2010) se ocupó de analizar las trayectorias de inserción de lxs jóvenes y los procesos por los cuales éstxs atraviesan antes de ingresar de modo estable al mercado laboral, tiempo en el que alternan períodos de desocupación, empleos precarios, pasantías y/o becas, etc. (Jacinto, 2000).

Por otra parte Macri (2013) analiza las trayectorias de jóvenes que habían empezado sus actividades laborales en la infancia remarcando que las trayectorias educativas y laborales de lxs jóvenes se caracterizan por ser diversas, no lineales y discontinuas.

Las características comunes de este grupo de investigaciones radican en el análisis de las trayectorias juveniles en el contexto de profundas transformaciones y el resquebrajamiento del modelo lineal de transición a la vida adulta. 
Dentro de esta categoría se encuentran las investigaciones de Otero y Córica (2017) que exponen resultados de una investigación en curso basada en un estudio sobre transiciones educativas y laborales de jóvenes de distintos sectores sociales, egresados de escuelas medias en Argentina de la última década. A partir de los itinerarios relevados las autoras afirman que se refuerza la tendencia hacia la continuidad educativa al finalizar la educación media. También indican la presencia de jóvenes que trabajan en simultaneo a estudiar y; entre los trabajadores se registra una alta variedad de ocupaciones juveniles distintiva en función del sector social y género. Si bien esta investigación no se ocupa del análisis de trayectorias estos autores dan luz al estudio ya que destacan las condiciones particulares que influyen en las rupturas y discontinuidades de los jóvenes dentro de las instituciones educativas y el mercado laboral.

Las investigaciones mencionadas ayudan a comprender principalmente que las biografías juveniles no pueden comprenderse al margen de las diversas transformaciones que ocurren en las esferas de la vida social ni por fuera de los marcos espacio temporales en los que se insertan.

\section{A modo de reflexión final}

Este artículo intentó brindar un recorrido específico por las categorías jóvenes, educación y trabajo a fin de aportar al conocimiento y reconstruir una sistematización que pueda servir como punto de partida para nuevas investigaciones sobre esta temática.

Como puede analizarse, los textos aquí mencionados describen, explican, argumentan, justifican y organizan la información existente. Sin lugar a dudas, como lo señalan muchas de estas investigaciones, la principal riqueza de cada uno consiste en la consideración del contexto micro social en el cual se llevaron a cabo.

Los análisis indagan en las particularidades, la multiplicidad de historias y realidades, entre contradictorias y afines, por las que atraviesan. De este modo, permiten reflexionar sobre cada situación particular, con semejanzas y diferencias de acuerdo al contexto social, económico y de cada momento histórico determinado. Por lo tanto, las experiencias juveniles pueden no ser similares a otras experiencias ya que éstas son una construcción social y personal. En este punto quizá radica la riqueza de los estudios sociales dado que sin perder de vista el actual contexto de globalización proponen el análisis de realidades particulares insertas en diversos contextos contemplando las particularidades de cada individuo y su entorno.

\section{Bibliografía}


ABAL MEDINA M.D, VÁZQUEZ M, J. (2004) Apropiarnos de los conocimientos que se construyen en nuestra práctica. Aportes a la sistematización de experiencias. Secretaría de Educación CTERA, Buenos Aires, Argentina.

AGULLÓ, T (1998) La centralidad del trabajo en el proceso de construcción de la identidad de los jóvenes: una aproximación psicosocial. Psicothema $\mathrm{N}^{\circ} 10$ Recuperado de: http://www.redalyc.org/articulo.oa?id=72710114

AISENSON, G., VALENUELA, V, CELEIRO, R, BAILAC, K. YLEGASPI, L. (2007) Representaciones de jóvenes que finalizan la escuela media sobre la escuela, el trabajo y el apoyo social. En Memorias de las Jornadas de Investigación en Psicología y Tercer Encuentro de Investigadores en Psicología del MERCOSUR, Volumen XIV. Universidad de Buenos Aires: Instituto de Investigaciones Facultad de Psicología, 205-208.

ANDRENACCI, L. Y SOLDANO, D (2006) Aproximación a las teorías de la política social a partir del caso argentino En Andrenacci, L. (comp.) Problemas de política social en la Argentina contemporánea. Los Polvorines: Prometeo, pp. 1780.

AUYERO (1993) Otra vez en la vía, Notas e interrogantes sobre juventud de sectores populares. Espacio Editorial, Buenos Aires.

BATTISTINI, O. (2004) (comp.), El trabajo frente al espejo. Continuidades y rupturas en los procesos de construcción identitaria de los trabajadores. Buenos Aires, Argentina: Prometeo.

BIALAKOWSKY, A. (2004) Identidades en el mundo del trabajo: Entre la implicación y la interpelación en Battistini, O., (comp.), El trabajo frente al espejo. Continuidades y rupturas en los procesos de construcción identitaria de los trabajadores. Buenos Aires, Argentina: Prometeo

BIGGART, A. (2002).Trayectorias fallidas, entre estandarización y flexibilidad en Gran Bretaña, Italia y Alemania Occidental." Revista de Estudios de Juventud, Jóvenes y transiciones a la vida adulta en Europa, No. 56, pp. 11-29.

BONDER, G. (1994) Mujer y Educación en América Latina: hacia la igualdad de oportunidades. Revista Iberoamericana de Educación. ISSN-e 16815653, ISSN 1022-6508, №6 (Ejemplar dedicado a: Género y Educación), pp. 9-48 BONO A. Y BULLONI, M.L (2008). Experiencias Laborales Juveniles. Los agentes telefónicos de los call centers offshore en Argentina. En Trabajo y Sociedad, Vol. IX. pp. $1-21$

BOURDIEU, P. (1990) Sociología y cultura, México: Grijalbo.

BOURDIEU, P. Y PASSWEON J.C. (1996) La reproducción. Elementos para una teoría del sistema de enseñanza. México: Fontamara. Trabajo original publicado en 1970. 
BRASLAVSKY,C. (1986). La juventud argentina entre la herencia del pasado y la construcción del futuro. Revista de la CEPAL. №29, pp.41-57.

( 1989.) "El caso argentino". Educación en la transición a la democracia.

Casos de Argentina, Brasil y Uruguay. Ed. C. Braslavsky, et al. Santiago de Chile: OREALC/UNESCO

(1995.) La Educación Secundaria en el contexto de los cambios en los sistemas educativos latinoamericanos, Revista Iberoamericana de Educación $N^{\circ} 9$ pp. 91 a 124.

BUSSO, M. Y GORBAN, D. (2004) Trabajando en el espacio urbano: La calle como lugar de construcciones y resignificaciones identitarias., en BATTISTINI, O: R. (coord.) El trabajo frente al espejo. Continuidades y rupturas en los procesos de construcción identitaria de los trabajadores. Buenos Aires, Argentina: Editorial Prometeo.

CASAL, J. (2006) Itinerarios y trayectorias. Una perspectiva de la transición de la escuela al trabajo. En Revista Trayectorias, vol. VIII, núm. 22, septiembrediciembre, 2006, pp. 9-20 Universidad Autónoma de Nuevo León Monterrey, Nuevo León, México

CASTEL,R (1997) Los riesgos de exclusión social en un contexto de incertidumbre. En Revista Internacional de sociología, Vol N², Nºxtra 1,2014. (ejemplar dedicado a: Procesos de exclusión social en un contexto de incertidumbre) pp. 15-24 CHAVES,M (2006) Informe Investigaciones sobre juventudes en Argentina: estado del arte en ciencias sociales. La Plata-Ciudad de Buenos Aires. Disponible http://www.comisionporlamemoria.org/investigacionyensenanza/pdf_biblioteca/I nforme-Investigaciones-sobre-juventudes-en-Argentina.pdf

CHAVES,M FUENTES S. Y VECINO L. (2016) Experiencias juveniles de la desigualdad. Fronteras y merecimientos en sectores populares, medios altos y altos, Buenos Aires, Argentina. Grupo Editor Universitario

CÓRICA, A. (2010). Lo posible y lo deseable. Expectativas laborales de jóvenes de la escuela secundaria. . Tesis de Maestría en Diseño y Gestión de Políticas y Programas Sociales - FLACSO. Buenos Aires.

(2012) Las expectativas sobre el futuro educativo y laboral de jóvenes de la escuela secundaria: entre lo posible y lo deseable. Última Década, n. 36, 71 -95. CORTI A.M. (2000) La vinculación entre educación y trabajo a finales del milenio. Revista Innovación Educativa 2000, № 10 pp.133-139.

DÁVILA LEÓN, O (2008) Trayectorias sociales juveniles. Ambivalencias y discursos sobre el trabajo. Valparaíso: Ediciones CIDPA e INJUV 
DÁVILA, O.; GHIARDO, F. Y MEDRANO, C. (2006), Los desheredados. Trayectorias de vida y nuevas condiciones juveniles, Valparaíso, Ediciones CIDPA (segunda edición aumentada.)

DUBET, F. (2006). El declive de la institución. Profesiones, sujetos e individuos en la modernidad. Barcelona, España: Genisa.

(2011) Repensar la justicia social. Contra el mito de la igualdad de oportunidades. Buenos Aires, Argentina: Ediciones Siglo Veintiuno

DURSI, C.; MILLENAAR, V. (2012), "De estudiante a trabajador. Estrategias y vínculos con el trabajo de jóvenes egresados del secundario en sus primeras inserciones", III reunión Nacional de Investigadores en Juventudes de Argentina, Red de Investigadores en Juventudes de Argentina (ReIJA), Universidad Nacional del Comahue, Viedma.

DUSCHATZKY, S. (1999) La escuela como frontera. Reflexiones sobre la experiencia escolar de jóvenes de sectores populares. Bs. As, Argentina: Paidós (2000) Tutelados y asistidos: programas sociales, políticas públicas y subjetividad. Buenos Aires: Paidós.

DUSSEL, I (2004) Desigualdades Sociales Y Desigualdades Escolares En La Argentina De Hoy. Algunas reflexiones y propuestas. Buenos aires, Argentina: FLACSO.

http://bibliotecavirtual.clacso.org.ar/ar/libros/argentina/flacso/dussel.pdf EPELÉ, M. (2010) Sujetar por la herida. Una etnografía sobre drogas, pobreza y salud. Buenos Aires, Argentina: Paidós

FEIJÓ M DEL C. Y CORBETTA S. (2004) Escuela y pobreza. Desafíos educativos en dos escenarios del Gran Buenos Aires, Bs As, Argentina: Instituto Internacional de Planeamiento de la Educación, IIPE-UNESCO.

FEIXA C. Y PORZIO L. (2004) Los estudios sobre culturas juveniles en España. Revista de Estudios de juventud. Número 64. Dedicado a De las tribus urbanas a las culturas juveniles. INJUVE.

GARCÍA, J (,2016) Bachilleratos Populares Y Estado: Relaciones Complejas y Dinámicas. En Publicar - Año XIV NºXXI// diciembre de 2016 - ISSN 0327-6627ISSN (en línea) 2250-7671.

POLOCK, G. (2002) Contingent Identities: Updating the transitional discourse, young. Sage Pub 10: 59

GLUZ, N. (2013) Alternativas escolares en movimientos sociales y procesos de subjetivación política. X Jornadas de Sociología. Facultad de Ciencias Sociales, Universidad de Buenos Aires, Buenos Aires. 
GRASSI E. Y DANANI C. (coord.) (2009); "El Mundo Del Trabajo Y Los Caminos De La Vida: Trabajar Para Vivir, Vivir Para Trabajar”. Buenos aires. Argentina: Editorial Espacio.

JACINTO, C (2016) Protección social y formación para el trabajo de jóvenes en la Argentina reciente. Entramados, alcances y tensiones. Buenos Aires, Argentina: Instituto de desarrollo Económico y Social

(2009) "Los dispositivos recientes de empleo juvenil: institucionalidades, articulaciones con la educación formal y socialización laboral ${ }^{e e}$, en Revista del Trabajo. Año $4 \mathrm{~N}^{\circ}$ 6. Ministerio de Trabajo, Empleo y Seguridad Social. Buenos Aires

(2010) "Introducción. Elementos para un marco analítico de los】 dispositivos de inserción laboral de jóvenes y su incidencia en las trayectorias”, en: Jacinto, C (Comp.) La construcción social de las trayectorias laborales de jóvenes: políticas, instituciones, dispositivos y subjetividades, Buenos Aires, Teseo/ IDES. pp.15-49.

(2006) Los protagonistas de la expansión de la educación secundaria. Anales de la educación común. Educación y trabajo. Tercer siglo, 2, (5). Buenos Aires: Dirección General de Cultura y Educación

(2000) Jóvenes vulnerables y políticas públicas de educación y empleo, mayo, Revista de estudios de juventud, n¹, nov. 2000, Buenos Aires, Dirección Nacional de Juventud, pp.103-121.

KESSLER, G. (2014) Controversias sobre la desigualdad. Argentina, 2003-2013”. Buenos Aires, Argentina: Fondo de Cultura Económica (2010) La sociedad argentina hoy. . Buenos aires, Argentina: Siglo XXI Editores

KROPFF, L. Y P. NÚÑEZ (2008). "Eje Acción, participación, opciones y estrategias políticas". En: Cháves, M. Juventudes en Argentina1. Hacia un estado del arte/2007. La Plata: Edulp

LA SERNA, C. (2010) La transformación del mundo del trabajo Representaciones, prácticas e identidades. Buenos Aires (Argentina): Fundación Centro de Integración, Comunicación, Cultura y Sociedad - CICCUS; Consejo Latinoamericano de Ciencias Sociales - CLACSO. Disponible en: http://biblioteca.clacso.edu.ar/clacso/becas/20120418051112/transformacion.pdf LONGO, M.E (2004) Jóvenes, precariedades y Sentidos del trabajo. 7Mo Congreso Nacional de estudios del Trabajo. Asociación Argentina de Especialistas en Estudios del Trabajo, Buenos aires, Argentina 
MACHADO PAIS, J. (2002) Laberintos de vida: paro juvenil y rutas de salida (jóvenes portugueses). Revista de Estudios de Juventud No56. Madrid, España: Injuve.

MACRI, M. (2013) Adolescentes, Estudiar y Trabajar, perspectivas en Argentina. Investigación en ciudad de Buenos Aires. Editorial Publicia-Saarbrucken, Deutschland/Alemania.I

(2010) Estudiar y trabajar: perspectivas y estrategias de los adolescentes. Buenos Aires, Argentina: La Crujía

MARGULIS, M. Y URRESTI, D. (2000) La segregación negada. Cultura y discriminación social en Buenos Aires (Buenos Aires: Biblos)

MARGULIS, M. (2002) La juventud es más que una palabra. Buenos Aires: Biblos.

MERADI, L. (2009) Alta Rotació. El trabajo precario de los jóvenes. Buenos aires, argentina: Tusquests Editores.,

MERKLEN, D. (2010) Pobres ciudadanos. Las clases populares en la era democrática (Argentina, 1983-2003). Buenos Aires: Gorla.

MIRANDA A. (2008). La nueva condición joven: educación, desigualdad y empleo. Buenos Aires, Argentina: Fundación Octubre

MIRANDA LÓPEZ, F. (2012) Los jóvenes contra la escuela. Un desafío para pensar las voces y tiempos para América Latina. Revista latinoamericana de educación comparada,

MONTES, N. (2010): «Principales rasgos de la educación secundaria desde la información estadística disponible». Propuesta Educativa $\mathrm{N}^{\circ} 34$. Disponible en línea: http://propuestaeducativa.flacso.org.ar

MORGADE, G. (2001) Aprender a ser mujer, aprender a ser varón. Buenos Aires: Novedades Educativas.

NOBILE, M. (2014) Emociones y vínculos en la experiencia escolar: el caso de las Escuelas de Reingreso de la Ciudad de Buenos Aires, (Tesis doctoral), Flacso Argentina.

NUÑEZ P.Y LITICHEVER L. (2015) Radiografías de la experiencia escolar: ser joven(es) en la escuela. 1a ed. Ciudad Autónoma de Buenos Aires: Grupo Editor Universitario, 2015

OTERO A Y CÓRICA A (2017) Población \& Sociedad [en línea], ISSN-L 0328 3445, Vol. 24 (2), 2017, pp. 33-64

OTERO, A. (2009) ¿El Trabajo En Transición? Apuestas, Valoraciones Y Construcciones Sobre La Educación Y El Trabajo Entre Jóvenes Argentinos De Hoy. Hallazgos de la tesis doctoral. Procesos de transición a la vida adulta: un estudio cualitativo con jóvenes argentinos. Doctorado de Ciencias Sociales FLACSO, Buenos Aires 
PÉREZ ISLAS, A. (2002) Jóvenes: Una evaluación del conocimiento: La investigación sobre juventud en México 1986-1999 (2 tomos). México: IMJ-CIEJ (edición corregida y aumentada)

PUIGRÓS, A Y DUSSEL I (1999) "Fronteras educativas en el fin de siglo: utopías y distopías en el imaginario pedagógico", en los límites de la educación formal: niños y jóvenes de fin de siglo, ed. por Adriana Puiggrós. Rosario, Homo Sapiens

REDONDO, P. (2004) Escuelas y pobreza. Entre el desasosiego y la obstinación. Buenos Aires, Argentina: Paidós.

REGUILLO CRUZ, R. (2000) Estrategias del desencanto. Emergencias de culturas juveniles. Buenos Aires, Argentina: Ed. Norma

ROMERO, P. (2012) Sociología de la empresa, el trabajo y las organizaciones. Un enfoque crítico. Madrid: Grupo 5, 325 pp.

SAINTOUT, F. (2010). «Los estudios socioculturales y la comunicación. Un mapa desplazado», artículo presentado en VIII Congreso Latinoamericano de Investigadores de la Comunicación - ALAIC São Leopoldo: Universidad de do Vale do Rio dos Sinos (UNISINOS).

(2006) Jóvenes. El futuro llegó hace rato. Comunicación y estudios culturales. La Plata: Ediciones de Periodismo y Comunicación.

SARAVÍ, G (2015) Juventudes fragmentadas. Socialización

(2010) Transiciones vulnerables. Juventud, desigualdad y exclusión en

México. CIESAS, México.

SENNET, R. (2010) El declive del hombre público. Buenos Aires, Argentina. Anagrama.

SILBA, M. (2006) Juventud, Identidad Y Experiencia: Las Construcciones Identitarias Populares Urbanas. Revista Questión; vol. 1, no 10. Universidad Nacional de La Plata http://sedici.unlp.edu.ar/handle/10915/33947

TENTI FANFANI, E. (2000), Una escuela para los adolescentes. Buenos Aires, Argentina: Editorial Losada.

TERIGI, F. (2007)Los desafíos que plantean las trayectorias escolares. Paper presentado en el III Foro Latinoamericano de Educación jóvenes y docentes. Fundación Santillana Buenos Aires, mayo 2007.

TIRAMONTI, G. (2009) “Una aproximación a la dinámica de la fragmentación del sistema educativo argentino. Especificaciones teóricas y empíricas” en Guillermina Tiramonti y Nancy Montes (comp.) La escuela media en debate. Problemas y perspectivas desde la investigación. Buenos Aires, Argentina: Manantial- Flacso.

TIRAMONTI, G. BRASLAVSKY, C. Y FILMUS, D. (1995), Las Transformaciones De La Educación En 10 Años De Democracia. Buenos Aires, Argentina. Flacso y Ed. Norma. 
URCOLA, M. (2003) Algunas apreciaciones sobre el concepto sociológico de la juventud. Rev. INVENIO. Universidad del Centro Educativo Latinoamericano. Rosario. Argentina

URRESTI, M. (2002) Culturas juveniles. En Beatriz Sarlo [et. al] compilado por Carlos Altamirano, Términos críticos de sociología de la cultura. Paidós, Buenos Aires. Kreimer, 1970; Grinberg, 1985, 1994; Senales 1980; Boimvaser, 2000; Grieco y Bavio, 1994; Lernoud, 1996; Escardo, Galende, Giberti, Invernizzi, 1996; De la Puente, Quintana, 1988)

VECINO, L. Y GUEVARA, B (2014) Sentidos de la experiencia escolar y vínculos intra e inter generacionales en cuatro escuelas secundarias de gestión estatal de la provincia de Buenos Aires, En IV Reunión Nacional de Investigadores en Juventud Argentina (RENIJA), llevado a cabo en FCJES(UNSL) Villa Mercedes, San Luis.

WORTMAN, A. (2001) Aproximaciones conceptuales y empíricas para abordar identidades sociales juveniles y consumos culturales en la sociedad del ajuste. Instituto de Investigaciones Gino Germani, Facultad de Ciencias Sociales, UBA ZATTARA, S Y SKOUMAL, G. (2008) "Chicas y chicos de sectores populares transitando la escuela media”, en Morgade, G. y G. Alonso (comps.), Cuerpos y sexualidades en la escuela, Buenos Aires, Paidós.

ZIEGLER, S. (2011). Entre la desregulación y el tutelaje: ¿hacia dónde van los cambios en los formatos escolares? En G. Tramonti (dir). Variaciones sobre la forma escolar. Límites y posibilidades de la escuela media (pp. 71-88) Rosario: Homo Sapiens Ediciones, Buenos Aires: FLACSO. 